\title{
Synchrotron Radiation X-Ray Multiple Diffraction in the Study of KDP Phase Transition Induced by Electric Field
}

\author{
A.O.dos Santos ${ }^{\mathrm{a}}$, R.V. Gelamo ${ }^{\mathrm{a}}$, B.B. Li ${ }^{\mathrm{a}}$, L.P. Cardoso ${ }^{\mathrm{a}}{ }^{*}$, M.A.R.Miranda ${ }^{\mathrm{b}}$, \\ M.A.M. Nogueira ${ }^{\mathrm{b}}$, C.M.R. Remédios ${ }^{\mathrm{b}}$, F.E.A.Melo ${ }^{\mathrm{b}}$, J.M. Sasaki ${ }^{\mathrm{b}}$, \\ L.H. Avanci ${ }^{\mathrm{c}}$, S.L. Morelhão ${ }^{\mathrm{c}}$ \\ ${ }^{\mathrm{a}}$ Instituto de Física Gleb Wataghin, Universidade Estadual de Campinas (UNICAMP), \\ C.P. 6165,13083-970 Campinas - SP, Brazil \\ ${ }^{\mathrm{b}}$ Dep. Física, Universidade Federal do Ceará (UFC), \\ C.P. 6030, 60455-760 Fortaleza - Ce, Brazil \\ ${ }^{\mathrm{c}}$ Instituto de Física, Universidade de São Paulo (USP), \\ C.P. 66318, 05315-970 São Paulo - SP, Brazil
}

Received: November 17, 2000; Revised: January 6, 2001

\begin{abstract}
In this work, the application of the X-ray multiple diffraction technique using synchrotron radiation at Laboratório Nacional de Luz Síncrotron (LNLS) to study KDP phase transition induced by electric field is discussed. A Huber three-axis $(\omega, \phi, 2 \theta)$ diffractometer mounted on a table which rotates around the incident beam allows to measure Renninger scans (RS) at the XRD station of LNLS using the adequate polarization for the experiments. Prior results indicated (080) as the best choice for the KDP primary reflection and the $(440)(\overline{4} 40)$ four-beam case for the secondary. Two Bragg-surface diffraction (BSD) reflections, where the secondary beam is propagated parallel to the sample surface, are involved in this multiple diffraction case. A RS interval of $3.3^{\circ}$ around $\phi=0^{\circ}$ position was measured while the electric field was increased up to $6 \mathrm{kV} / \mathrm{cm}$. At this value, a phase transition was observed and the secondary peaks became narrower and changed their RS position indicating possible occurrence of a metastable ferroelectric phase.
\end{abstract} field

Keywords: X-ray multiple diffraction, synchrotron radiation, phase transition, electric

\section{Introduction}

Among several ferroelectric materials which contain hydrogen bonds, potassium dihydrogen phosphate (KDP) is probably the most investigated. At a temperature of $122 \mathrm{~K}$, the $\mathrm{KDP}\left(\mathrm{KH}_{2} \mathrm{PO}_{4}\right)$ crystals undergo a ferroelectric phase transition, where occurs the lowering of the crystal symmetry from the tetragonal I42d phase to the orthorhombic Fdd2 phase. As a result the crystal lattice becomes polarized along the c-axis. Several works reporting the stability of both phases as a function of the hydrostatic pressure and low-intensity DC electric fields were published ${ }^{1-4}$. It presents ferroelectric properties above $\mathrm{T}=123 \mathrm{~K}$ in a tetragonal symmetry with 4 formulas of $\mathrm{KH}_{2} \mathrm{PO}_{4}$ per unit cell (spatial group I42d) with lattice parameters: $\mathrm{a}=\mathrm{b}=7.453 \AA$ and $\mathrm{c}=6.974 \AA$. Below this transition temperature, the crystal presents an orthorrombic symmetry with 8 formulas of $\mathrm{KH}_{2} \mathrm{PO}_{4}$ per unit cell, in a

e-mail: cardoso@lua.ifi.uncamp.br

Trabalho apresentado no $14^{\circ}$ CBECIMAT , Águas de São Pedro, Dezembro 2000 spatial group Fdd2 with lattice parameters: $a=10.530 \AA$, $\mathrm{b}=10.44 \AA$ and $\mathrm{c}=6.90 \AA$.

Recently $^{5}$, X-ray diffraction has been used to study electric field induced structural changes in deuterated KDP and the authors claim to have obtained a very good agreement when comparing their results with the temperature induced changes reported in the literature.

The X-ray multiple diffraction technique can, in principle, provide three-dimensional information on the analyzed lattice and also, enough sensitivity to detect subtle changes in lattice geometry of single crystals when submitted to external stimuli, such as an applied electric field. The technique was used as a new probe to study the effect of an external electric field in the case of two organic crystals which allowed the determination of the piezoelectric coefficients ${ }^{6,7}$. 
Multiple diffraction arises when an incident beam simultaneously satisfies the Bragg law for more than one set of lattice planes within a crystal. A set of planes called primary $\left(h_{p}, k_{p}, \lambda_{p}\right)$, usually parallel to the sample surface, is aligned to diffract the incident beam. The sample is then rotated ( $\phi$-axis) around the primary reciprocal lattice vector and several secondary planes $\left(\mathrm{h}_{\mathrm{s}}, \mathrm{k}_{\mathrm{s}}, \lambda_{\mathrm{s}}\right)$ with arbitrary orientation also diffract. The coupling reflection $\left(h_{p}-h_{s}\right.$, $\left.\mathrm{k}_{\mathrm{p}}-\mathrm{k}_{\mathrm{s}}, \lambda_{\mathrm{p}}-\lambda_{\mathrm{s}}\right)$ is responsible to establish the intensity interaction among the primary and the secondary reflections. The record of the $\mathrm{I}_{\text {primary }} v s . \phi$ is called Renninger scan $(\mathrm{RS})^{8}$ and it shows peaks (Umweganregung) when the relative strengths of the reflections participating in a multiple diffraction process interfere constructively or dips (Aufhellung) in the converse case. Extremely asymmetric cases of the multiple diffraction phenomenon called Bragg surface diffraction (BSD) ${ }^{9}$ appear when the secondary beam is propagated parallel to the sample surface and are very useful to study crystallographic perfection at the surface/interface sample ${ }^{10}$.

The symmetry plays an important role in the multiple diffraction phenomenon and the Renninger scan shows symmetry mirrors ${ }^{11}$ established by the primary vector symmetry and also by the symmetry of the reciprocal lattice points when they enter and leave the Ewald sphere. This makes the RS very sensitive to small changes in the crystalline lattice since it should promote symmetry changes. Normally, it is easier to work with weak (or forbidden by the space group) primary reflections since this enhances the peak/background ratio in the RS. Therefore, it facilitates the sample alignment, the exact determination of the peak position and the maximum intensity. Unfortunately, this is not possible for all multiple diffraction applications.

The multiple diffraction technique becomes more powerful when uses the unique advantages of the synchrotron radiation as the linear polarization and high intensity. Then, the objective of this work is to show that by adding a Huber three-axis diffractometer in the geometry of the XRD station, high resolution conditions can be obtained for multiple diffraction experiments with enough sensitivity to study KDP phase transition induced by external electric field.

\section{Experimental}

The samples used in X-ray MD experiment using synchrotron radiation were cut from good optical quality and good crystalline perfection grown by slow temperature technique in parallelepipeds of dimensions of $5 \mathrm{~mm} \times 7 \mathrm{~mm}$ $\mathrm{x} 2 \mathrm{~mm}$ and oriented by X-ray diffraction. The good quality of the crystal was determined by performing X-ray synchrotron radiation rocking curve for 080 reflection where the FWHM is about 5.7 seconds of arc as shown in Fig. 1. For X-ray diffraction experiment, we cut from the same block a crystal with dimensions of $0.87 \mathrm{~mm}$ x $3 \mathrm{~mm}$ x $5 \mathrm{~mm}$.

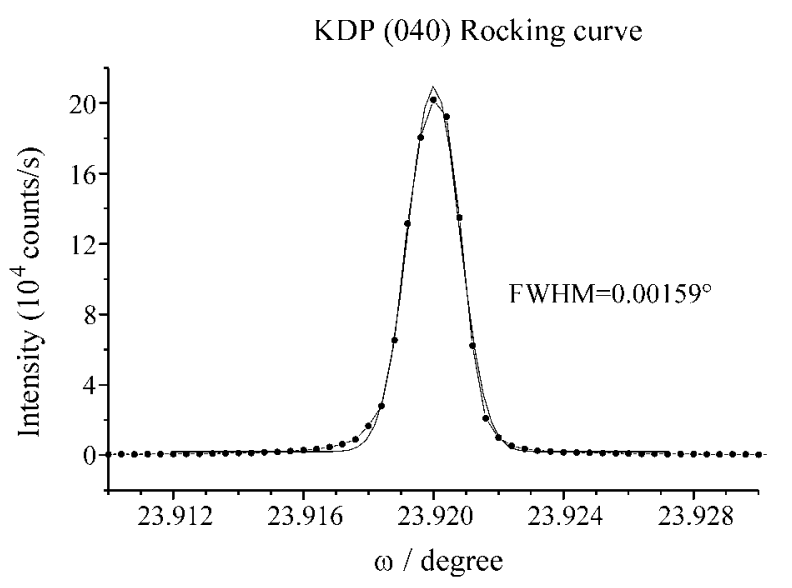

Figure 1. Rocking curve obtained at XRD station of the LNLS. The experimental points are presented with the gaussian fitting just to provide a better visualization.

Renninger scans were carried out at LNLS using $\lambda=1.3394 \AA$ in a geometry which consists of two-channel cut $\mathrm{Si}(111)$ crystals as monochromator. A Huber three-axis diffractometer was fixed on a table which provides rotation ( $\chi$ axis) around the primary beam direction from 0 to $90^{\circ}$, allowing polarization measurements. This $\omega: \phi$ diffractometer mounted at the XRD station of the LNLS also provides high resolution Renninger scans with step sizes of $0.0002^{\circ}$ and $0.0005^{\circ}$ in $\omega$ and $\phi$ axes, respectively. It was built to allow the study of single crystals by X-ray diffraction through conventional techniques of rocking curves $(\omega)$ and reciprocal space mapping ( $\omega / 2 \theta$ scan), together with those non-conventional techniques like Renninger scans $(\phi$ scan) and BSD mapping ( $\omega: \phi$ scan). High resolution rotating elements $(\omega, \phi$ and $2 \theta)$ were assembled to permit the use of the goniometer in both vertical and horizontal directions. Then, diffraction experiments using the LNLS synchrotron radiation at XRD station can be performed taking advantage of the possibility of varying the linear polarization from $0^{\circ}$ ( $\sigma$ polarization) up to $90^{\circ}$ ( $\pi$ polarization) with respect to the diffractometer incidence plane. In KDP measurements, we have used the above-mentioned method to obtain just umweganregung peaks in the RS by changing the primary linear polarization ${ }^{12,13}$. The $(080)$ primary intensity was monitored while the polarization was changed to the position of the primary minimum value $\left(0^{\circ}\right)$ and the result is shown in Fig. 2. For multiple diffraction, this enhances the secondary peak intensity, particularly the strong BSD ones, to provide easy sample alignment and RS with good resolution.

The electric field was generated by a variable voltage, low current dc power supply and applied to the samples via wires running from the power supply to small bolts attached to the metal tabs of the sample holder. Pieces of a conduc- 


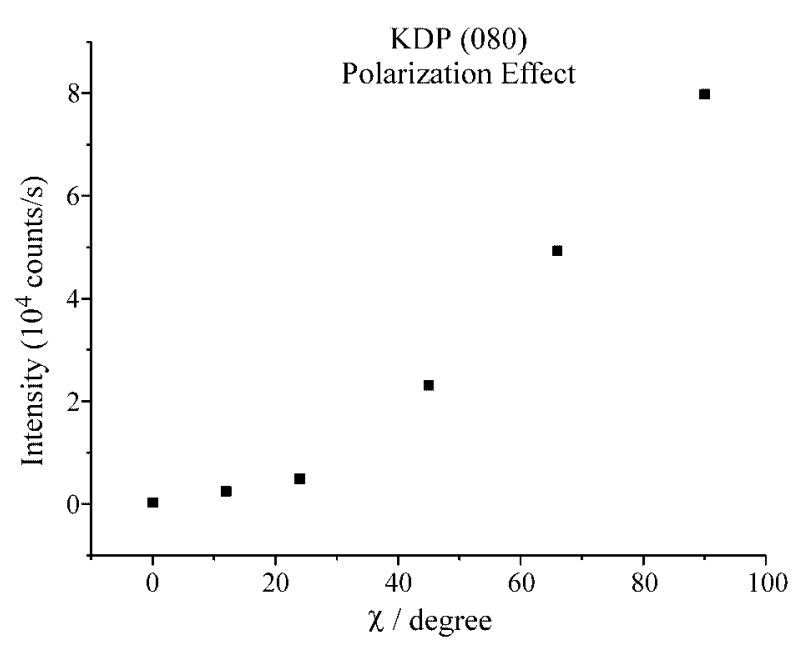

Figure 2. Effect of the variation of the synchrotron linear polarization with respect to the diffractometer incidence plane to the (080) KDP intensity.

tive sponge kindly supplied by SGL Carbon Meitingen were positioned between the tabs and sample to improve the electrical contact. The sample holder itself was made of an insulating material so the electric field applied parallel to the dipolar $\mathrm{b}$ axis, passed only through the sample.

\section{Results and Discussion}

In order to investigate KDP structural changes induced by an external electric field $\mathrm{E}$ exploratory measurements using a normal powder diffractometer were performed. A Rigaku diffractometer with Mo as X-ray source was used to carried out theta-2theta measurements for (220) and (440) reflections under electric field applied to [001] direction up to $12 \mathrm{kV} / \mathrm{cm}$. Figure 3 shows the results for the integrated intensity $v s$. electric field. One can clearly observe abrupt changes in intensity around $6 \mathrm{kV} / \mathrm{cm}$ possibly due to phase transition. The good penetration of the X-ray beam allows to get diffraction from deeper planes therefore, avoiding the non-uniformity of electric field on surface. This preliminary result was important to support our idea of using the high resolution synchrotron radiation X-ray multiple diffraction.

Figure 4 shows the Renninger scan portion around the $\phi=0^{\circ}$ symmetry mirror for the primary reflection $(080)$ of KDP obtained in the LNLS set up. It should be noted in this figure that the (080) primary intensity now is weak since the incident beam polarization was changed to $0^{\circ}$ (see Fig. 2) with the availability of the new geometry developed at the XRD station of the LNLS. The scan appears indexed and shows the strong 4-beam case (000)(080)(440)(4 40) exactly at mirror position for $\mathrm{E}=0 \mathrm{kV} / \mathrm{cm}$. It should be noted that, in this case with (080) primary, both secondary

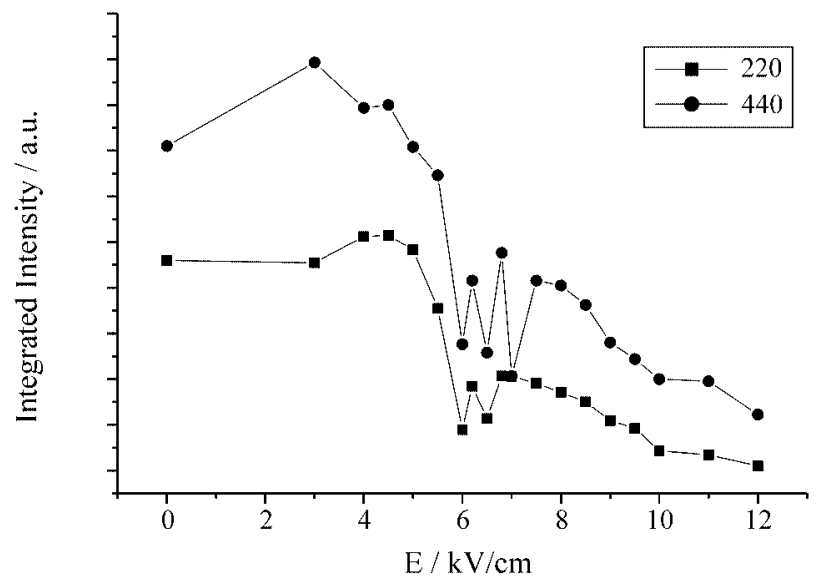

Figure 3. KDP integrated intensity of (220) and (440) as a function of the electric field (E[001]) showing the induced phase transition at $\mathrm{E}=6 \mathrm{kV} / \mathrm{cm}$ for $\mathrm{T}=28^{\circ} \mathrm{C}$.

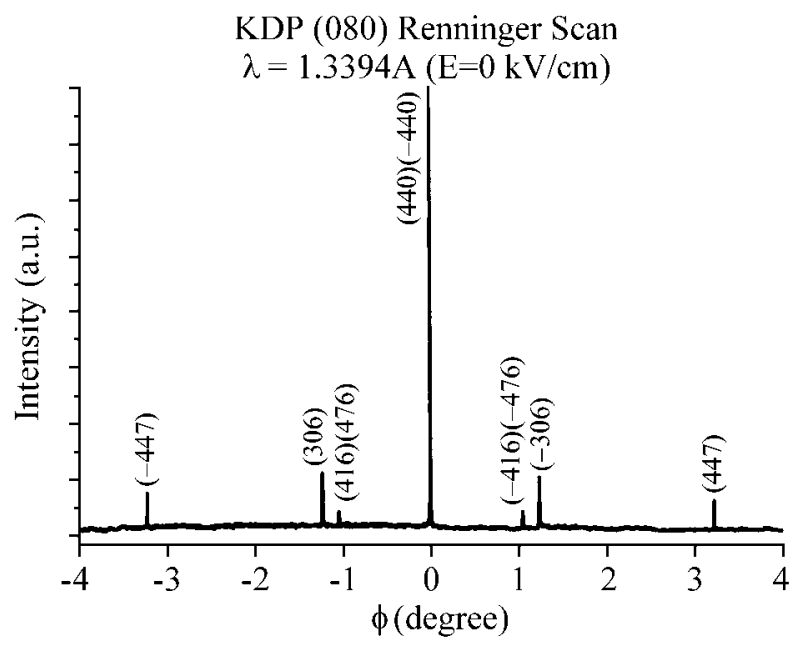

Figure 4. Region around $\phi=0^{\circ}$ symmetry mirror of the KDP (080) Renninger scan obtained at XRD station.

reflections (440) and (4 40) stand for two BSD peaks. Therefore, the particular angular position of this 4-beam case becomes very important when the electric field is applied since it can be used as a standard angular reference in the Renninger scan. Another weaker BSD peak (447) is also observed in this Figure close to $\phi=3.2^{\circ}$ besides two other visible peaks: (000)(080)(06) three-beam Bragg case and the (000)(080)(416)(4 76) four-beam case.

Figure 5 depicts the effect of the electric field in the KDP Renninger scan. For each E value, the primary reflection, now the (880) following the extinction rules for phase transition tetragonal to orthorrombic symmetry, was realigned in order to provide a good Renninger scan. The (440) secondary reflection is still a BSD case. The measured interval includes the four above-mentioned peaks for 


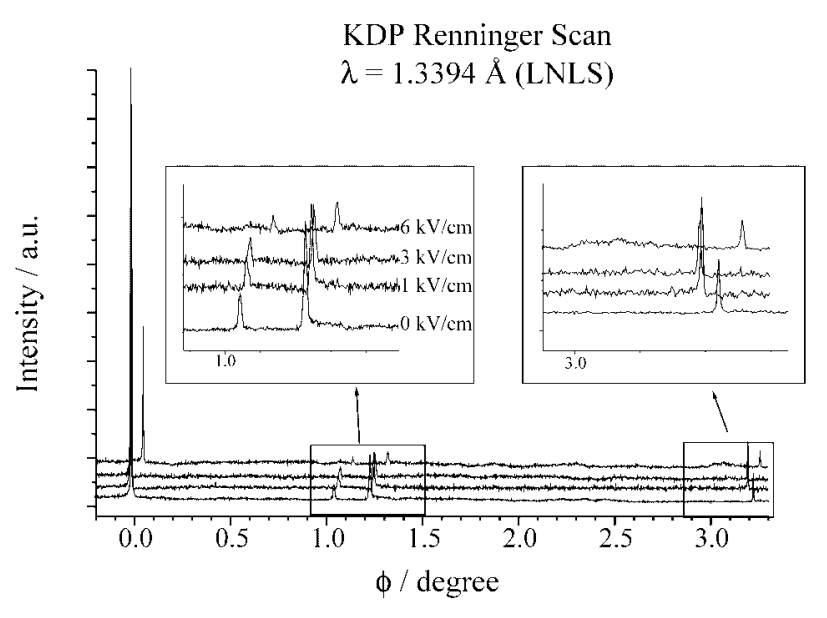

Figure 5. Effect of the electric field in the KDP (080) Renninger scan. Insets show details of the peak shift.

different $E$ values. The two insets show details of the peaks appearing around $\phi=1.2^{\circ}$ and $3.3^{\circ}$. The angular positions of these peaks have changed as the electric field was applied and the phase transition close to $\mathrm{E}=6 \mathrm{kV} / \mathrm{cm}$ is clearly visible. This $\mathrm{E}$ value is the same obtained from the results shown in Fig. 3. It confirms that the modifications in the XRD station geometry allows for multiple diffraction experiments with the possibility of choosing almost any primary reflection providing an umweganregung Renninger scan. Moreover, the set up has enough resolution to detect electric field induced phase transition in KDP samples.

Finally, it should be pointed out that we were also able to confirm the irreversibility of the KDP phase transition after the paraelectric phase has changed to the metaestable ferroelectric one. The position of the new phase four-beam BSD peak around $0^{\circ}$ remained unchanged even when the electric field was turned off by 2 days after the transition has occurred.

\section{Conclusions}

It has been shown in this work that the modifications in the geometry of the XRD station of the LNLS by adding the Huber three axis $(\omega, \phi$ and $2 \theta)$ diffractometer makes possible to obtain Renninger scans (and also mapping of BSD peaks not shown in this work) with high resolution besides the normal two-beam diffraction experiments. During the measurements, the linear polarization with respect to the diffractometer incidence plane can be varied between 0 and $90^{\circ}$ causing drastic changes in the intensity ratios of the multiple diffracted beams. This allows one to work with just umweganregung Renninger scans by choosing, in principle, any desired primary reflection. Furthermore, we have shown that the phase transition induced by electric field in KDP samples was used to show the sensitivity and high resolution of the synchrotron radiation X-ray multiple diffraction.

\section{Acknowledgments}

The authors wish to thank the valuable help of the staff of the LNLS in the performance of these experiments. We also acknowledge the financial support of the Brazilian Agencies FAPESP and CNPq.

\section{References}

1. Itoh, K.; Matsubayashi, T.; Nakamura, E.; Motegi, H. Journal of Physics Society of Japan, v. 39, p. 843, 1975.

2. Melo, F.E.A.; Serra, K.C.; Souza, R.C.; Moreira, S.G.C.; Mendes-Filho, J.; Moreira, J.E. Brazilian Journal of Physics, v. 22, p. 95,1992.

3. Morosin, B.; Samara, G. Ferroelectrics, v. 3, p. 49, 1971.

4. Pearcy, P.S.; Samara, G. Physical Review B, v. 8, p. 2033, 1973.

5. van Reeuwijk, S.J.; Puig-Molina, A.; Graafsma, H. Physical Review B, v. 62, n.10, p. 6192, 2000.

6. Avanci, L.H.; Cardoso, L.P.; Girdwood, S.E.; Pugh, D.; Sherwood, J.N.; Roberts, K.J. Physical Review Letters, v. 81, n. 24, p. 5426, 1998.

7. Avanci, L.H.; Cardoso, L.P.; Sasaki, J.M.; Girdwood, S.E.; Roberts, K.J.; Pugh, D.; Sherwood, J.N. Physical Review B, v. 61, n. 10, p. 6506, 2000.

8. Renninger, M. Z Kristallogr., n. 106, p. 14, 1937.

9. Hayashi, M.A.; Morelhão, S.L.; Avanci, L.H.; Cardoso, L.P.; Sasaki, J.M.; Kretly, L.C.; Chang, S.L. Applied Physics Letters, v. 71, n. 18, p. 2614, 1997.

10. Avanci, L.H.; Hayashi, M.A.; Cardoso, L.P.; Morelhão, S.L.; Riesz, Ferenc, Rakennus, K.; Hakkarainen, T. Journal of Crystal Growth, n. 188, p. 220, 1998

11. Chang, S.L. Multiple Diffraction of X-Rays in Crystals, Springer Verlag, Ser. Solid-State Sci., v. 50, Berlin, Heidelberg, New York, 1984.

12. Stetsko, Y.P.; Jeretschke, H.J.; Huang, Y.S.; Chao, C.H.; Chen, C.K.; Chang, S.L. Acta Crystallographica, v. A56, p.394, 2000.

13. Morelhão, S.L.; Avanci, L.H. Acta Crystallographica, v. A57, 2001 (in press).

FAPESP helped in meeting the publication costs of this article 